\title{
Experimental and clinical analysis of the use of asymmetric vs symmetric polyethylene inserts in a mobile bearing total knee arthroplasty
}

\author{
Gianluca Castellarin $^{\mathrm{a}}$, Edoardo Bori ${ }^{\mathrm{b}, *}$, Bernardo Innocenti ${ }^{\mathrm{b}}$ \\ ${ }^{a}$ II Unit Orthopaedic Department, Ospedale di Suzzara, Mantova, Italy \\ ${ }^{\mathrm{b}}$ BEAMS Department, Université Libre de Bruxelles, Bruxelles, Belgium
}

\section{A R T I C L E I N F O}

\section{Keywords:}

TKA

Mobile bearing

Asymmetric insert

Symmetric insert

Insert congruency

Biomechanics

Kinematics

\begin{abstract}
A B S T R A C T
Purpose: This study compared the effects of symmetric and asymmetric designs for mobile bearing polyethylene insert for total knee arthroplasty (TKA), both clinically and biomechanically through experimental cadaver tests. Methods: 303 patients implanted with a mobile bearing TKA were retrospectively analyzed up to 2-year follow-up with relative scores. The same femoral and tibial components were used for all the patients; 151 patients received a Symmetric Design (SD) insert and 152 an Asymmetric Design (AD). A biomechanical experimental test was performed to improve the comprehension of the clinical results, analyzing passive squat on 5 cadaveric knee specimens: internal-external rotations of femur and tibial insert respect to the tibia tray were analyzed in native and implanted configurations (with both symmetrical and asymmetrical inserts for each specimen).

Results: After surgery, patients' average flexion improved from $105^{\circ}$ (with preoperative extension deficit of $5^{\circ}$ ), to $115^{\circ}$ (SD-group) and $120^{\circ}$ (AD-group) at the 2-year follow-up. There was no postoperative extension deficit. AD-group presented better ability to perform certain routines and wasn't affected by any pain, while anterolateral pain was reported in some SD-group patients. The experimental tests returned no statistically relevant difference in tibio-femoral flexion-extension and internal-external rotations among all the three configurations tested; a statistically significant difference is found for insert-tray internal-external rotations between SD and AD configurations; in details, the $\mathrm{AD}$ insert showed insert-tray angles comparable to the ones found for femoral component-tibial tray, while the SD insert returned lower angular values.

Conclusion: Clinically and biomechanically, an asymmetric mobile bearing insert could represent a valid alternative to symmetric mobile bearing insert.

Level of evidence: III, Case-control study Retrospective comparative study.
\end{abstract}

\section{Introduction}

Total knee arthroplasty (TKA) is a highly successful and reproducible treatment for patients having serious knee joint issues, with more than 600,000 surgeries performed each year in the USA. ${ }^{1}$ The goal of knee replacement is to recover normal functionality and kinematics in patients and, in order to best meet each individual's demand, several solutions for TKA designs are currently available on the market. However, no absolute guidelines are available to select the best matching solution for each situation; a potential reason for this lies in the limited amount of clinical and biomechanical evidence-based justifications on the effects of the different design features characterizing the various available products. $^{2-7}$
Mobile-bearing (MB) TKA designs offer the theoretical advantage of increased implant conformity, and therefore contact area, minimizing polyethylene contact stress and therefore wear. ${ }^{8-10}$ The insert rotation, achieved through the tibial tray-polyethylene bearing articulation, theoretically minimizes the transfer of torsional stresses to the fixation interfaces that are present in case of fixed bearing TKA prosthetic designs. ${ }^{9,10}$ Despite most of the MB inserts currently available are symmetric, among the different designs on the market GENUS MB (Adler Ortho, Cormano, Milan, Italy) also proposes an insert with asymmetric design for the TKA system GENUS LS. Analyzing in detail this design, it's to highlight that only the proximal surface of the asymmetric insert differs from the symmetric one for its geometry and congruencies; on the other hand, size, dimensions and the other features are identical among

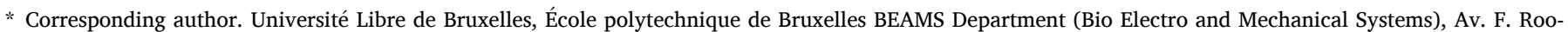
sevelt, 50 CP165/56, 1050, Bruxelles, Belgium.

E-mail address: edoardo.bori@ulb.be (E. Bori).
} 


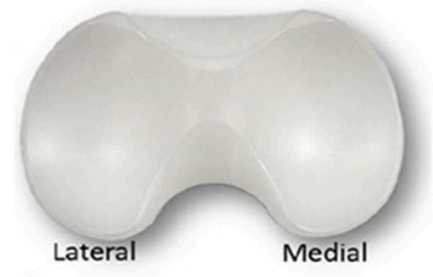

a

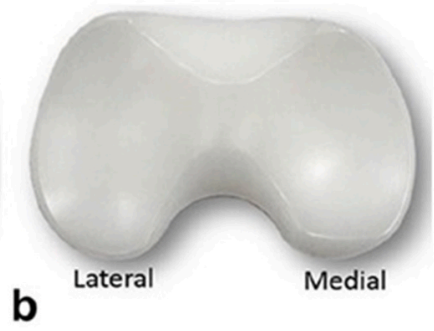

Fig. 1. Overview of the insert design analyzed in this study: Symmetrical Design (SD) (a) and Asymmetrical Design (AD) (b).

the two inserts in order to allow its coupling with the tibial component.

In order to evaluate and understand the actual differences and benefits of the use of an asymmetric MB insert compared to a symmetric one, this work aimed to investigate both inserts from a clinical and a biomechanical point of view, following the principles of evidence-based medicine. In detail, a 2-years retrospective clinical study of 303 patients was performed; following, biomechanical tests were performed using a Motion-Capture system to monitor knee kinematics during passive squat movements on 5 knee joint cadaveric specimens in native configuration and with both inserts. The biomechanical results were then analyzed in relation with the retrospective follow-up results in order to justify and understand the findings.

The importance of this study resides in the improvement of the investigation on the benefits of an asymmetric solution for mobile bearing TKA, achieved through clinical and biomechanical experimental evidences. The results could be used to help understanding patient's dissatisfaction after a TKA, which is usually related to replaced joint kinematics and kinetics not faithfully respecting the physiological ones. ${ }^{1,2,4,5,8-14}$

\section{Materials and methods}

\subsection{Clinical study}

Every patient gave his/her written informed consent to have his/her clinical records later used for this retrospective study. In the period 2012-15, the first author [X.X.] performed 303 consecutive primary MB TKA in 303 patients [109 males (36\%), average 70.2y (range 56-87y), 171 right knees (56\%)]. All procedures performed in this study involving human participants were in accordance with the ethical standard of the institutional and/or national research committee and with the 1964 Helsinki Declaration and its later amendments or comparable ethical standards.

In all the procedures, a Genus MB (Adler Ortho, Milan, Italy) was implanted. Genus MB received an ODEP 5A* rating (www.odep.org.uk). All TKAs were cemented and patella was not resurfaced. Medial parapatellar approach was adopted for all patients. For all patients, the same designs of the femoral and the tibial components were used, while two possible selections were available for the polyethylene insert: the symmetric design (SD) and the asymmetric design (AD) (See Fig. 1). The first 151 patients [35\% males, 57\% right knee, average 70.3y (range $56-87 y)$ ] were implanted with the SD insert, while the AD solution was adopted for the following 152 patients [( $37 \%$ males, 56\% right knee, average 70.1y (range 56-83y)]. For the group undergoing the SD insert the pre-operative average flexion was $103.0^{\circ}\left(93^{\circ}-112^{\circ}\right)$, while for the patients of the $\mathrm{AD}$ insert the preoperative average flexion was $105.0^{\circ}$ $\left(95^{\circ}-110^{\circ}\right)$.

The PCL was preserved in all patients, and ligaments release was avoided whenever possible by the use of a Minimally Invasive alignment device (EMAS, Extra-Medullary Alignment system) offered by the manufacturer. ${ }^{15-17}$ In detail, the EMAS device allows for femoral component positioning and joint balancing without requiring the use of intramedullary rods, avoiding violation of the intramedullary canal, which can potentially enable the incidence of fat embolism and perioperative blood loss or femoral fractures risk in the case of osteoporotic bone. ${ }^{2,15,18,19}$

Particular care was taken to minimize the tibial bone cut, in order to preserve bone integrity. In detail, the $10 \mathrm{~mm}$ insert (the smallest thickness provided by the manufacturer) was used in $88 \%$ of the cases: as SD insert, the $10 \mathrm{~mm}$ insert was used on 127 patients, the $12 \mathrm{~mm}$ on 20 patients and the $14 \mathrm{~mm}$ for the remaining 4 patients; for the $\mathrm{AD}$ group, 135 patients received the $10 \mathrm{~mm}$ insert and the remaining 17 patients received the $12 \mathrm{~mm}$ insert; no patient received the $14 \mathrm{~mm}$ insert. This choice was made in agreement with the study of Berend et al. $2010^{20}$ in which the author found that bearings with thickness greater than $16 \mathrm{~mm}$ were associated with higher failure rates at midterm to long-term follow-up. Patients belonging to both groups then followed the same post-op rehabilitation protocol.

Clinical and radiographic evaluations were performed at six months, one year and two years after the operation by the first author [X.X.] together with an experienced surgeon belonging to his staff. Standing antero-posterior, lateral, and Merchant radiographs were evaluated according to the system of the Knee Society for bone cement interface radiolucency, polyethylene wear, any change in the position of the component, alignment, and osteolysis.

The Knee Society score ${ }^{21}$ and the Oxford knee score ${ }^{11,22}$ were calculated for all patients pre-operatively, at six months, at one year and at two years post-operatively. The active range of motion was determined with the use of a standard clinical goniometer.

\subsection{Biomechanical study}

For the biomechanical part of the study, 5 cadaveric knee joint specimens with no history of musculoskeletal problems were analyzed.

Each specimen was fixed to a specifically designed frame, equipped with 10 OptiTrack Flex 3 Cameras (NaturalPint Inc., Corvallis, OR United States). ${ }^{23}$ Following the Motion Capture protocol already used in previous studies, ${ }^{7,24-26}$ several sets of markers were applied to 3D-printed stands rigidly attached to femur, tibia and insert (when present); sets of biomechanical landmarks ${ }^{27,28}$ were then determined on tibia and femur. Anatomic bone coordinate systems and joint rotations were calculated based on the Grood \& Suntay coordinate system. ${ }^{29}$ To define internal-external rotation of the femoral frontal plane, the line joining the medial and lateral condyle centers was used instead of the posterior condyles tangent line. ${ }^{7,27}$ The kinematic test was then performed by applying a passive flexion-extension movement (of approximately $90^{\circ}$ amplitude) to the knee joint by moving the tibia with the femur fixed, while recording the process. ${ }^{7,24-26}$ For the 5 specimens, each configuration analyzed was tested and recorded three times for the sake of repeatability.

The tests were completed firstly in native configuration and then, after having performed the TKA operation (performed by the first author following the same surgical approach adopted in the clinical study), repeated in prosthetic configuration with both $\mathrm{SD}$ and $\mathrm{AD}$ inserts. The outputs were then analyzed (as done in previous studies ${ }^{7,24-26}$ ) in order to extract the relative kinematics of the different rigid bodies through the movement; for each specimen, the results from all the configurations were then compared to evaluate the differences in insert internal-external rotation on the tibial component according to the two designs.

\subsection{Statistical analysis}

To check the proper population size for the study a Power analysis was performed. In details, an alpha error of 0.05 (universally accepted) was considered, with a beta error of 0.2 (giving a power of $80 \%$ ) and an effect size of $0.35 .^{30}$ The results of the power analysis, performed with $\mathrm{G}^{*}$ Power 3.1.2, ${ }^{31,32}$ results in a total sample of 272 patients. The 


\section{Knee Society Score}

120

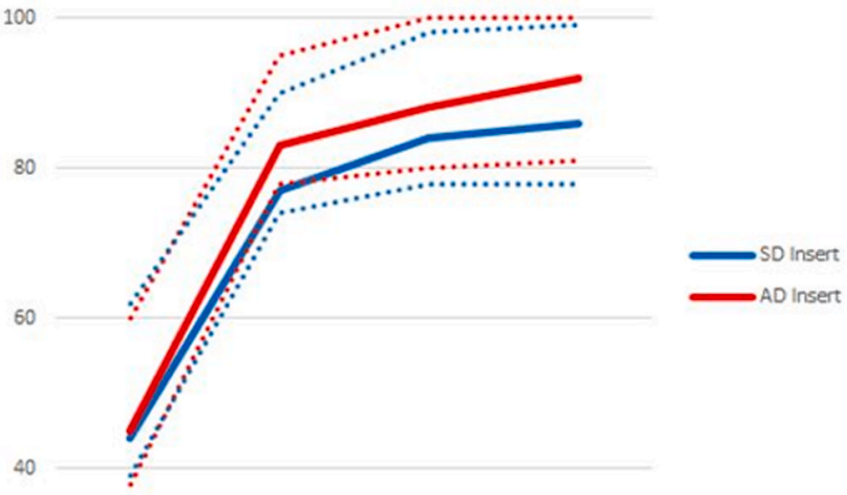

20

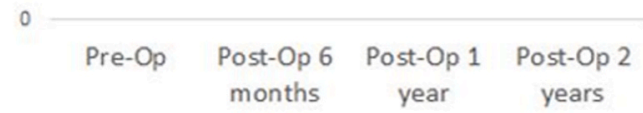

Fig. 2. Knee Society Score at Pre-Op and at the different Follow-ups, represented as Average (continuous line) and Maximum-Minimum (dotted lines) for the two designs.

selection of circa 150 patients for group, with a total sample size of 303 , can therefore be considered adequate.

For the statistical analysis, the $t$-test was used for the numerical variables while the Wilcoxon-Mann test was used for ordinal variables. A preliminary Shapiro-Wilk test was performed to check the normality of the data collected. Null hypotheses of no difference were rejected if two-sided p-values were less than 0.05. All the statistical analysis were performed using Matlab (MATLAB and Statistics Toolbox Release 2012b, The MathWorks, Inc., Natick, Massachusetts, United States).

\section{Results}

\subsection{Clinical study}

The two analyzed groups presented no significant differences in terms of demographic characteristics, and all surgeries were completed without any relevant intra-operative or post-operative complication, hence successfully.

At the 2-year follow-up, the patients of the SD-group returned an average flexion of $118^{\circ}$ (range $=105^{\circ}-135^{\circ}, \mathrm{SD}=5.245$ ), while the ADgroup reported an average flexion up to $125^{\circ}$ (range $115^{\circ}-135^{\circ}$, SD $=$ 5.393), with no extension deficit; statistically significant difference is found $(\mathrm{p}<0.05)$. Initial antero-lateral pain, which nonetheless tended to diminish and disappear after some months, was reported by $30 \%$ of the patients implanted with SD insert; no pain affected instead the patients with the $\mathrm{AD}$ insert, who additionally showed greater selfconfidence during the movements.

Both Knee Society Score ${ }^{21}$ and Oxford Knee Score ${ }^{11,22}$ were then compared to evaluate the clinical performance and degree of satisfaction after the TKA; the graphs in Figs. 2 and 3 show the trends and values of the scores taken at the different follow-ups, expressed as average,

\section{Oxford Score}

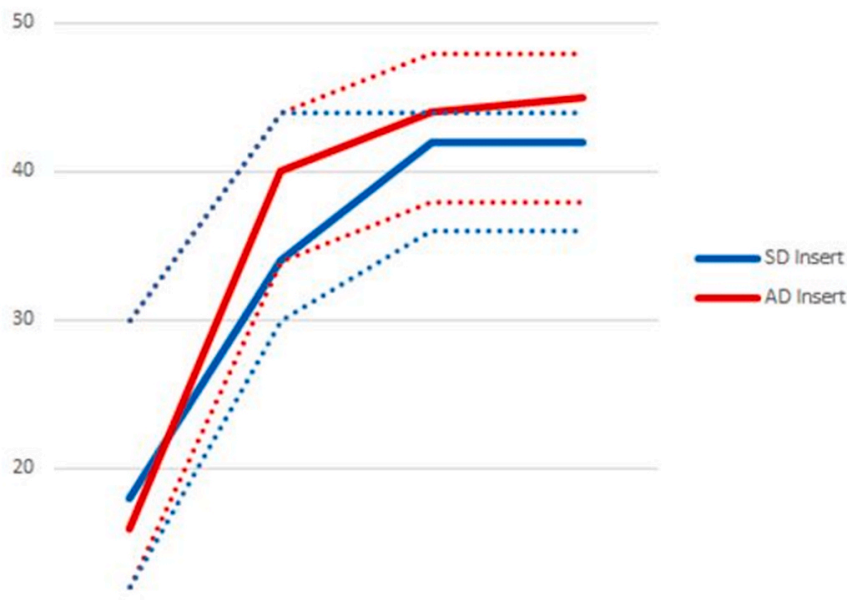

10

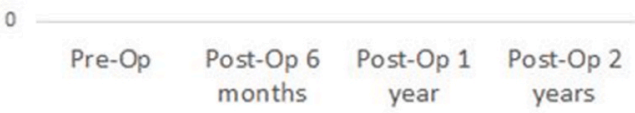

Fig. 3. Oxford Score at Pre-Op and at the different Follow-ups, represented as Average (continuous line) and Maximum-Minimum (dotted lines) for the two designs.

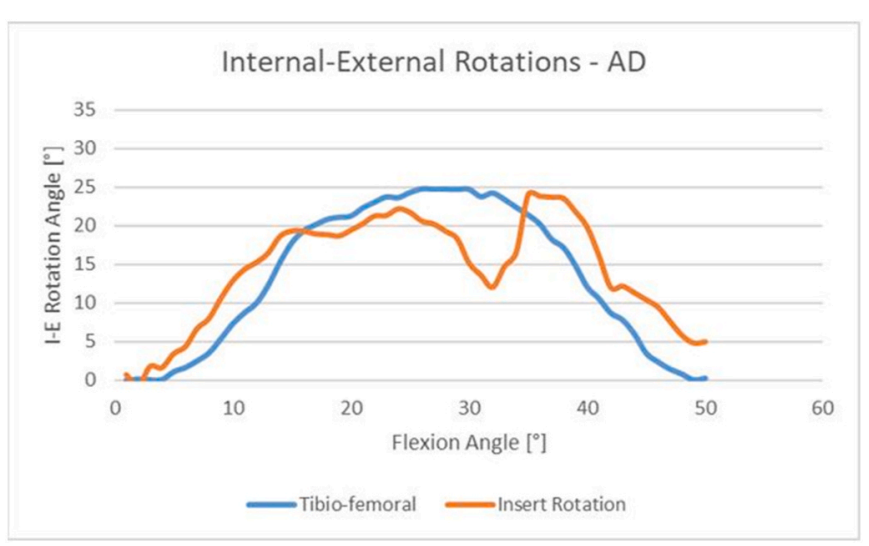

Fig. 4. Internal-External Rotations in degrees of Femur (in blue) and Asymmetrical Design Insert (in orange) with respect to the Tibia.

minimum and maximum. Both the scores increase significantly at 2-year follow-up. Moreover, the values of both scores are statistically higher in the $\mathrm{AD}$ insert with respect to the SD insert $(\mathrm{p}<0.05)$.

\subsection{Biomechanical study}

The biomechanical results showed that the $\mathrm{AD}$ insert is able, during the flexion-extension movement, to rotate on the tibial tray following the internal-external femoral rotation in a better way if compared to the SD (as shown for a single specimen in Figs. 4 and 5, range and trend are in fact almost equivalent to the values obtained between femur and tibia, meaning almost zero rotation between femur and insert). 


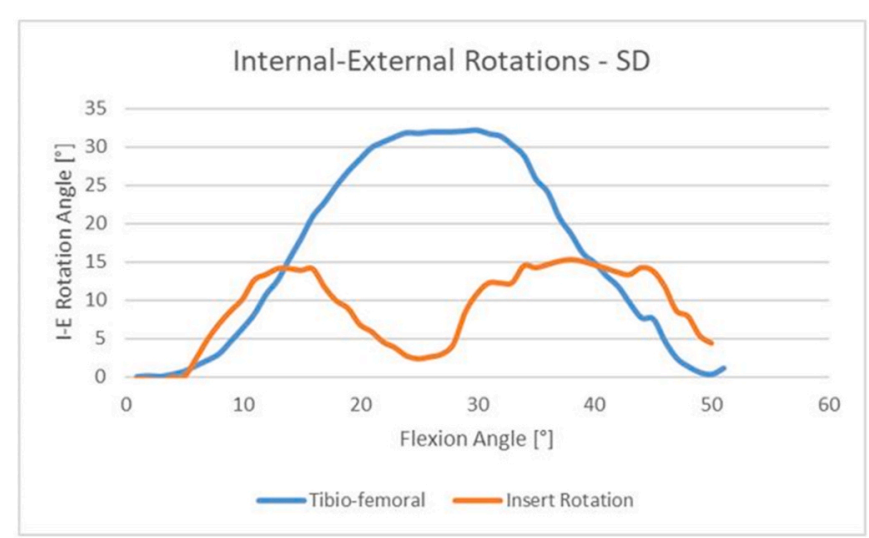

Fig. 5. Internal-External Rotations in degrees of Femur (in blue) and Symmetrical Design Insert (in orange) with respect to the Tibia.

The standard insert, on the other hand, returns lower values of internal-external rotation compared to the tibio-femoral ones; it is to be noted, though, that similar patterns in the initial phase of the flexion can be found (see Fig. 4) and this can be due to an initial congruence between insert and femur, followed by a relative sliding then causing the difference in the rotation values obtained (see Fig. 5).

The graph in Fig. 6 reports, for each configuration, the excursion of the angles of flexion and internal-external rotation measured during the motion task, expressed as average and standard deviation. The values of tibio-femoral internal-external rotation resulted to be similar among all the configurations, regardless of the presence of an implant or of the insert design.

\section{Discussion}

The biomechanical study showed that the ranges for the rotation between tibia and insert are in agreement with the clinical-numerical study previously carried out and published ${ }^{15,33}$; the $\mathrm{AD}$ insert design is furthermore able to internally/externally rotate in agreement with the femur, thus limiting the relative motion between the two. This factor is in agreement with the findings of the previous study [15], which stated that the stresses found in case of $\mathrm{AD}$ design were lower if compared to the ones in SD: indeed, the higher compliance of the insert with femoral internal-external rotation enables contact areas between the two components to be broader and to maintain this situation along the whole motion, resulting then in the lower stresses. This result is due to the design of the AD insert which, while maintaining high congruence in the medial sector, allows the lateral condyle to slide on its plateau; this peculiarity allows the prosthesis to follow the variation of the physiological internal-external rotation axis (which occurs during the flexion extension movement analyzed), consequently leading to the minimization of rotation between the $\mathrm{AD}$ insert and the femoral component. Therefore, the SD insert shows similar trend in rotation for the beginning of flexion while returning lower angles in the following phases, due to its congruence on both medial and lateral sides: indeed, this factor, constraining also the lateral condyle, prevents the insert to follow the physiological variation of the femoral internal-external rotation axis and then leads the femur to slide on the insert, lowering the contact areas then worsening the stress distribution.

It should be noted that the tibio-femoral internal-external rotations values are in the order of $20^{\circ}$ and have been found in case of passive movement, performed manually by the operator; these results are therefore in agreement with the experimental tests performed by. ${ }^{34-40}$

Clinical and biomechanical results are then in agreement: it is remarkable how both MB solutions are able to achieve good results for the patients in terms of luxation or instability, which were not present even in case of a totally not congruent lateral compartment (as the one in the $\mathrm{AD}$ inserts); agreement is also found tibio-femoral rotations, as the global kinematics outputs are similar for the two solutions. However, there is a difference in how the global kinematics is determined and

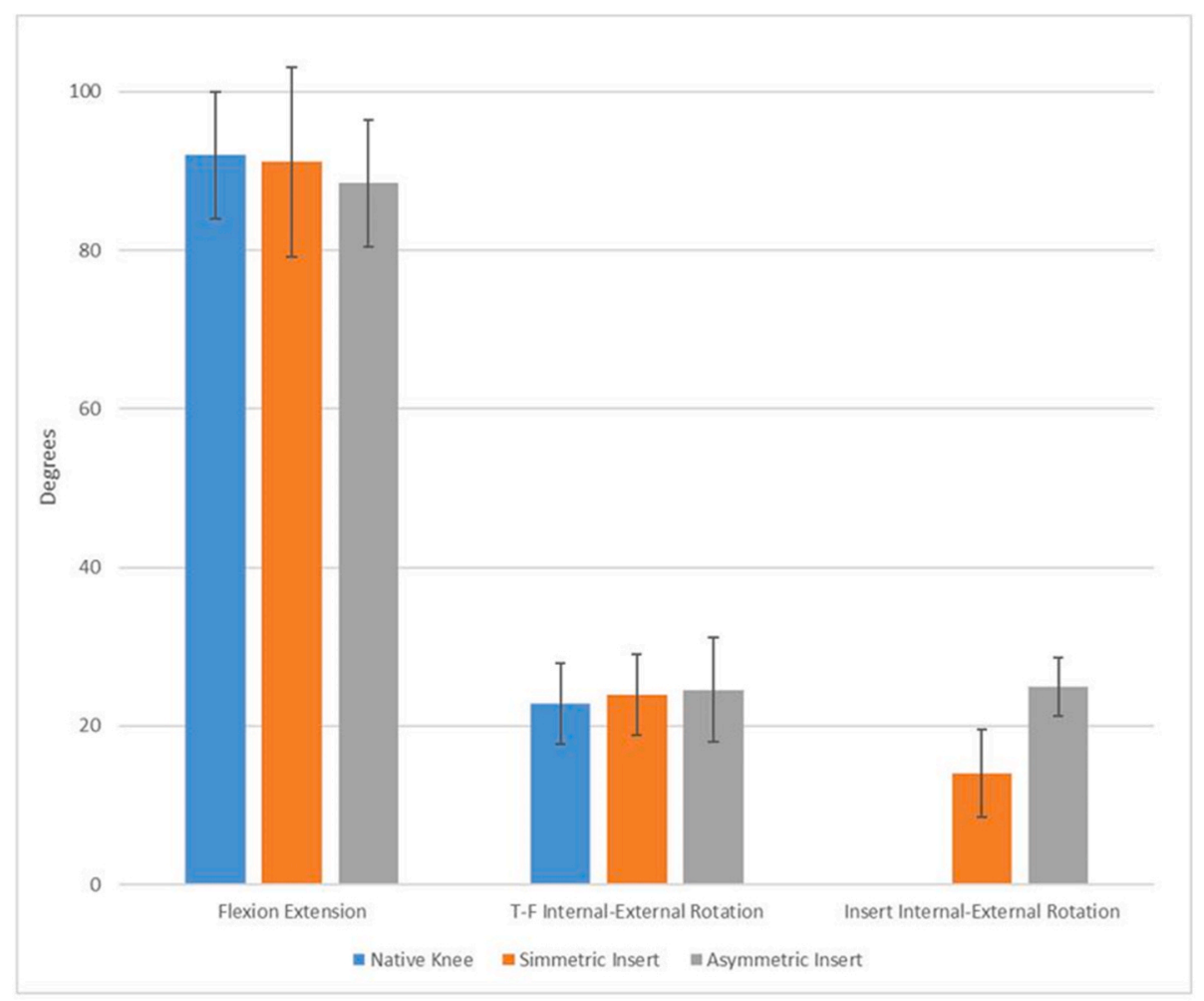

Fig. 6. Excursion of tibio-femoral flexion angles, femoral internal-external rotation angles on the tibia and internal-external rotation angles of the insert on the tibia; each value is expressed in degrees, as average and standard deviation, for the different configurations. 
hence, thanks to the lateral sliding solution, better performances are achieved and the patient is more self-confident with the implant.

Another factor to consider is that the differences in shear and compression stresses of the two designs are extremely important: the increase in motion of the $\mathrm{AD}$ design represents an advantage for the stress reduction towards the tibial bone, specifically for what concerns the shear stress. The decrease in stress will then bring to better performance of the tibial component, in terms of cement stability and lift-off: the lesser the stress coming from the femoral component, the less stress at the tibial component-bone interface. This outcome is thus in agreement with the findings of the previous study, ${ }^{15}$ and it can be a reason for many patients implanted with the $\mathrm{AD}$ to feel less pain rather than the ones with the SD.

The present clinical study showed then that an $\mathrm{AD}$ mobile bearing insert is able to provide better performances (in terms of KSS and Oxford scores) also at 2-year post-op follow-up if compared to a standard SD insert; accordingly, the clinical evidence showed that the patients operated with the $\mathrm{AD}$ had a higher satisfaction level and a better ability to perform physical activities as shown by the better Oxford score seen for that group.

It is nonetheless to be added that the particular surgical technique employed (not involving any femoral canal invasion, guaranteeing PCL preservation and minimal, if any, soft-tissue release) was probably an additional beneficial contribution to the patients' positive post-op performances. ${ }^{2,15,18,19}$

It is to highlight that all the surgeries, both for the clinical and the biomechanical sections of the present study, were performed by the same surgeon, namely the first author [X.X.], in the same hospital center; one of the main limitations of the work consists in the fact that only one model of prosthesis was analyzed (despite featuring two different insert designs). It is nonetheless to highlight that the results of this study are a consequence of the features of the prosthesis, so their significance can be broadened to the whole category of symmetric and asymmetric inserts.

Another limitation is then related to the biomechanical tests, that were performed on 5 specimens only, considering a passive squat and analyzing kinematics; additional tests, also involving muscle forces and acquiring kinetics, can be done in order to further increase the amplitude of the research.

\section{Conclusions}

The experimental biomechanical analysis justifies the clinical findings: despite the kinematics is comparable for the two designs, the $\mathrm{AD}$ solutions showed higher agreement with femoral internal-external rotation, thus resulting as being more suitable to be cemented, also avoiding lift-off issues, and inducing less pain.

Asymmetric mobile bearing insert is shown to provide better clinical performances (KSS and Oxford scores) at 2-years follow up, compared to a standard symmetric insert design.

The results of this comparative study demonstrate that, both clinically and biomechanically, an asymmetric mobile bearing insert could represent a more valid solution rather than symmetric mobile bearing insert; surgeons should then consider this option as a valid possibility in the prosthesis selection for a patient.

\section{References}

1 Kurtz S, Ong K, Lau E, et al. International survey of primary and revision total knee replacement. Int Orthop. 2011;35(12):1783.

2 Li Y, Wu Q, Ning G, et al. No difference in clinical outcome between fixed- and mobile-bearing TKA: a meta-analysis. Knee Surg Sports Traumatol Arthrosc. 2014;22 (3):565-575.

3 Luyckx T, Didden K, Vandenneucker H, et al. Is there a biomechanical explanation for anterior knee pain in patients with patella alta? Influence of patellar height on patellofemoral contact force, contact area and contact pressure. J Bone Joint Surg. 2009;91(3):344-350.
4 Smith H, Jan M, Mahomed N, et al. Meta-analysis and systematic review of clinical outcomes comparing mobile bearing and fixed bearing total knee arthroplasty. J Arthroplasty. 2011;26(8):1205-1213.

5 Smith T, Ejtehadi F, Nichols R, et al. Clinical and radiological outcomes of fixedversus mobile-bearing total knee replacement: a meta-analysis. Knee Surg Sports Traumatol Arthrosc. 2010;18(3):325-340.

6 Soenen M, Baracchi M, De Corte R, et al. Stemmed TKA in a femur with a total hip arthroplasty: is there a safe distance between the stem tips? J Arthroplasty. 2013;28 (8):1437-1445

7 Victor J, Labey L, Wong P, et al. The Influence of muscle load on tibio-femoral knee kinematics. J Orthop Res. 2010;28(4):419-428.

8 Carothers JT, Kim RH, Dennis DA, et al. Mobile-bearing total knee arthroplasty: a meta-analysis. J Arthroplasty. 2011;26(4):537-542.

9 Hopley CD, Crossett LS, Chen AF. Long-term clinical outcomes and survivorship after total knee arthroplasty using a rotating platform knee prosthesis: a meta-analysis. J Arthroplasty. 2013;28(1):68-77.

10 Kwak JY, Jeong JH, Lee SH, et al. Comparison of the clinical outcomes after total knee arthroplasty with the LCS rotating platform mobile bearing knee system and the PFC sigma RP-F mobile bearing knee system. Clin Orthop Surg. 2012;4(4):256-262.

11 Dawson J, Fitzpatrick R, Murray D, et al. Questionnaire on the perceptions of patients about total knee replacement. J Bone Joint Surg Br. 1998;80(1):63-69.

12 Oh KJ, Pandher DS, Lee SH, et al. Meta-analysis comparing outcomes of fixedbearing and mobile-bearing prostheses in total knee arthroplasty. $J$ Arthroplasty. 2009;24(6):873-884.

13 Pianigiani S, Labey L, Pascale W, et al. Knee kinetics and kinematics: what are the effects of TKA malconfigurations? Knee Surg Sports Traumatol Arthrosc. 2016;24(8): 2415-2421.

14 Wen Y, Liu D, Huang Y, et al. A meta-analysis of the fixed-bearing and mobilebearing prostheses in total knee arthroplasty. Arch Orthop Trauma Surg. 2011;131 (10):1341-1350.

15 Castellarin G, Pianigiani S, Innocenti B. Asymmetric polyethylene inserts promote favorable kinematics and better clinical outcome compared to symmetric inserts in a mobile bearing total knee arthroplasty. Knee Surg Sports Traumatol Arthrosc. 2019;27 (4):1096-1105.

16 Castellarin G, Cimino V. A simple technique to perform total knee replacement without violating the femoral canal: early clinical results on a cohort of 303 patients. Eur J Orthop Surg Traumatol. 2020;30(8):1377-1382.

17 Castellarin G. A novel surgical technique to perform total knee arthroplasty in patients with inaccessible femoral medullary canal. J Orthop. 2019;27(19):102-105.

18 Caillouette JT, Anzel SH. Fat embolism syndrome following the intramedullary alignment guide in total knee arthroplasty. Clin Orthop Relat Res. 1990;251:198-199.

19 Yau WP, Ng TP, Chiu KY. Unusual complication associated with femoral intramedullary alignment guide in total knee arthroplasty. J Arthroplasty. 2001;16 (2):247-249.

20 Berend ME, Davis PJ, Ritter MA, et al. "Thicker" polyethylene bearings are associated with higher failure rates in primary total knee arthroplasty. J Arthroplasty. 2010;25 (6): $17-20$.

21 Scuderi GR, Bourne RB, Noble PC, et al. The new knee society knee scoring system. Clin Orthop Relat Res. 2012;470(1):3-19.

22 Murray DW, Fitzpatrick R, Rogers K, et al. The use of the Oxford hip and knee scores. J Bone Joint Surg Br. 2007;89(8):1010-1014.

23 Arcidiacono GIB, Macrì A, et al. Design of a measurement system for the estimation of the knee kinematics. Int J Mech Eng Technol. 2018;9(2):866-875.

24 Heesterbeek PJ, Labey L, Wong P, et al. A new spacer-guided, PCL balancing technique for cruciate-retaining total knee replacement. Knee Surg Sports Traumatol Arthrosc. 2014;22(3):650-659.

25 Heyse TJ, El-Zayat BF, De Corte R, et al. UKA closely preserves natural knee kinematics in vitro. Knee Surg Sports Traumatol Arthrosc. 2014;22(8):1902-1910.

26 Victor J, Van Glabbeek F, Vander Sloten J, et al. An experimental model for kinematic analysis of the knee. J Bone Joint Surg Am. 2009;91(Suppl 6):150-163.

27 Victor J, Van Doninck D, Labey L, et al. How precise can bony landmarks be determined on a CT scan of the knee? Knee. 2009;16(5):358-365.

28 Innocenti B, Salandra P, Pascale W, et al. How accurate and reproducible are the identification of cruciate and collateral ligament insertions using MRI? Knee. 2016; 23(4):75-581.

29 Grood ES, Suntay WJ. A joint coordinate system for the clinical description of threedimensional motions: application to the knee. J Biomech Eng. 1983;105:136-144.

30 Leppin J, O'Sullivan P, Winston K. Effect size - large medium and small. Perspect Med Educ. 2016;5(6):347-349.

31 Faul F, Erdfelder E, Buchner A, et al. Statistical power analyses using G*Power 3.1: tests for correlation and regression analyses. Behav Res Methods. 2009;41(4): 1149-1160.

32 Faul F, Erdfelder E, Lang AG, et al. *Power 3: a flexible statistical power analysis program for the social, behavioral, and biomedical sciences. Behav Res Methods. 2007;39(2):175-191.

33 Arnout N, Vanlommel L, Vanlommel J, et al. Post cam mechanics and tibiofemoral kinematics: a dynamic in vitro analysis of eight posterior stabilized total knee designs. Knee Surg Sports Traumatol Arthrosc. 2015;23(11):3343-3353.

34 Blankevoort L, Huiskes R, de Lange A. Helical axes of passive knee joint motions. J Biomech. 1990;23:1219-1229.

35 Bull AM, Earnshaw PH, Smith A, et al. Intraoperative measurement of knee kinematics in reconstruction of the anterior cruciate ligament. J Bone Joint Surg Br. 2002;84:1075-1081.

36 Grood ES, Stowers SF, Noyes FR. Limits of movement in the human knee. Effect of sectioning the posterior cruciate ligament and posterolateral structures. J Bone Joint Surg Am. 1988;70:88-97. 
37 Hill PF, Vedi V, Williams A, et al. Tibiofemoral movement 2: the loaded and unloaded living knee studied by MRI. J Bone Joint Surg Br. 2000;82-B:1196-1198.

38 Iwaki H, Pinskerova V, Freeman MAR. Tibiofemoral movement 1: the shapes and relative movements of the femur and tibia in the unloaded cadaver knee. $J$ Bone Joint Surg Br. 2000;82-B:1189-1195.
39 Most E, Axe J, Rubash H, et al. Sensitivity of the knee joint kinematics calculation to selection of flexion axes. J Biomech. 2004:37:1743-1748.

40 Wilson DR, Feikes JD, Zavatsky AB, et al. The components of passive knee movement are coupled to flexion angle. J Biomech. 2000;33:465-473. 